\title{
Evolution of structural and optical properties of ion-beam synthesized GaAsN nanostructures
}

\author{
X. Weng, S. J. Clarke, W. Ye, S. Kumar, and R. S. Goldman ${ }^{\text {a) }}$ \\ Department of Materials Science and Engineering, University of Michigan, Ann Arbor, \\ Michigan 48109-2136
}

A. Daniel and R. Clarke

Applied Physics Program, University of Michigan, Ann Arbor, Michigan 48109-1120

J. Holt, J. Sipowska, and A. Francis

Department of Chemistry, University of Michigan, Ann Arbor, Michigan 48109-1055

V. Rotberg

Department of Nuclear Engineering and Radiological Sciences, University of Michigan, Ann Arbor, Michigan 48109-2104

(Received 14 February 2002; accepted for publication 9 July 2002)

\begin{abstract}
We have investigated the evolution of structural and optical properties of GaAsN nanostructures synthesized by $\mathrm{N}$ ion implantation into epitaxial GaAs, followed by rapid thermal annealing. Transmission electron microscopy and x-ray diffraction indicate the formation of nanometer-sized crystallites with lattice parameters close to those of pure zincblende GaN. The average crystallite size increases with annealing temperature while the size distribution is self-similar and the volume fraction remains constant, suggesting a coarsening process governed by Ostwald ripening. These GaAsN nanostructures exhibit significant photoluminescence in the near infrared range. The apparent lowering of the fundamental band gap is likely due to the incorporation of a small amount of As in GaN. (C) 2002 American Institute of Physics. [DOI: 10.1063/1.1504177]
\end{abstract}

\section{INTRODUCTION}

For many group III-N alloys, significant energy band gap bowing has been predicted and observed. ${ }^{1-5}$ For example, for $\sim 1 \%$ atomic nitrogen added to GaAs, the band gap is reduced by $\sim 200 \mathrm{meV}^{3,5,6}$ Similarly, the introduction of $\sim 1 \%$ arsenic into $\mathrm{GaN}$ has reduced the band gap by $\sim 700$ $\mathrm{meV}^{4}$ The resulting narrow gap nitride semiconductors are promising for a wide range of applications, including longwavelength light-emitters and detectors, high-performance electronic devices, and high efficiency solar cells. ${ }^{1}$ In the case of GaAs: N, a consequence of the large N-As size difference is a predicted limited miscibility on the anion sublattice, which leads to the formation of phase separationinduced alloy nanostructures. ${ }^{7-11}$ To date, only a few percent nitrogen has been incorporated substitutionally in GaAs:N synthesized by conventional epitaxial methods. ${ }^{3}$

An alternative approach is to implant nitrogen into GaAs and other group III-V compounds, followed by an appropriate annealing sequence. To date, conflicting results have been reported regarding the formation of GaNAs alloys in $\mathrm{N}$ ion implanted-plus-annealed GaAs. For example, dilute GaNAs alloys have apparently been synthesized by multiple energy $\mathrm{N}$ ion implantations into GaAs substrates, followed by thermal annealing. ${ }^{12-16}$ This process was intended to produce $>0.3-\mu \mathrm{m}$-thick GaNAs layers with nearly uniform nitrogen concentration. ${ }^{12-16}$ In these studies, reductions of the band gap energy, observed by either photoluminescence (PL)

a) Author to whom correspondence should be addressed; electronic mail: rsgold@engin.umich.edu spectroscopy or photoreflectance, were attributed to the formation of dilute GaNAs alloys. On the other hand, in a related ion-beam-synthesis study, an apparent shortwavelength photoluminescence emission was observed and attributed to the formation of wurtzite (WZ) GaN. ${ }^{17}$ Furthermore, one earlier study considered the nanometer-scale structural evolution of the $\mathrm{N}$ ion-implanted-plus-annealed GaAs, without mention of the optical properties. ${ }^{18}$ In the latter study, both zincblende (ZB) and randomly oriented WZ GaN crystallites in a crystalline GaAs matrix were observed. ${ }^{18} \mathrm{In}$ addition, for the highest $\mathrm{N}$ dose studied, a continuous layer of randomly oriented $\mathrm{WZ} \mathrm{GaN} \mathrm{nanocrystals} \mathrm{was} \mathrm{produced.}$

To date, most reports of ion-beam-synthesized GaAs: N have focused either on the optical properties of the implanted substrates, without consideration of the nanometer-scale structure, or on the structural evolution, without mention of the optical properties. Thus, the relationship between the nanometer-scale structural evolution and the optical properties of ion-beam-synthesized GaAs: $\mathrm{N}$ is not well understood. Therefore, in this article, we report an investigation of the evolution of the nanometer-scale structural and optical properties of GaAsN synthesized by nitrogen ion implantation into GaAs, followed by rapid thermal annealing (RTA). High-resolution transmission electron microscopy (HRTEM) reveals nanometer-sized crystallites surrounded by matrices which do not apparently possess the same degree of long range order. Electron and $\mathrm{x}$-ray diffraction indicate crystallite lattice parameters similar to those of $\mathrm{ZB} \mathrm{GaN}$. The average size of these crystallites increases with annealing temperature while the size distribution is self-similar and the crystal- 
lite volume fraction remains constant. Together, these results suggest that the crystallite coarsening process is governed by Ostwald ripening which occurs during annealing. Furthermore, these GaAsN nanostructures exhibit significant photoluminescence in the near infrared range. The apparent lowering of the fundamental band gap of the GaN-rich nanostructures is likely due to the incorporation of As in GaN.

The article is organized as follows. In Sec. II we describe the methods for synthesizing and characterizing the nanostructures, including ion implantation, rapid thermal annealing, x-ray diffraction, transmission electron microscopy, and photoluminescence spectroscopy. In Sec. III, the structural evolution and correlations between the nanometer-scale structural and optical properties are presented and discussed. Conclusions are given in Sec. IV.

\section{EXPERIMENTAL PROCEDURES}

For these investigations, $\sim 2-\mu \mathrm{m}$-thick GaAs films grown by molecular beam epitaxy on (001) GaAs were implanted with nitrogen ions at an incident ion energy of 100 $\mathrm{keV}$ and a dose of $5 \times 10^{17} \mathrm{~cm}^{-2}$. During implantation, the substrate temperature was maintained at $300^{\circ} \mathrm{C}$. According to PROFILE code simulations ${ }^{19}$ (with sputtering coefficients determined using the TRIM $\operatorname{code}^{20}$ ), these conditions were predicted to result in the largest near-surface nitrogen retention with least surface sputtering. In particular, these conditions were predicted to lead to a maximum $\mathrm{N}$ concentration of $2.1 \times 10^{22} \mathrm{~cm}^{-3}$, located $\sim 80 \mathrm{~nm}$ below the surface. A retained nitrogen dose of $\sim 3 \times 10^{17} \mathrm{~cm}^{-2}$ was confirmed via nuclear reaction analysis using the reaction ${ }^{14} \mathrm{~N}(d, \alpha){ }^{12} \mathrm{C} .{ }^{21}$ Some of the implanted samples were subsequently RTA in argon gas at 750,800 , or $850^{\circ} \mathrm{C}$ for $30 \mathrm{~s}$. Since the RTA was performed without a surface cap, some decomposition of the GaAs surface may have occurred.

Cross sectional TEM specimens were prepared using conventional mechanical polishing, followed by argon ion milling at $77 \mathrm{~K}$. TEM imaging and electron diffraction were carried out in a JEOL 4000EX operating at $400 \mathrm{kV}$ and a JEOL 2010FX operating at $200 \mathrm{kV}$. Both high-resolution and dark-field diffraction contrast images were obtained in the JEOL 4000EX. Selected area diffraction (SAD) patterns were obtained in the JEOL 4000EX, using apertures which select regions with $\sim 0.14$ and $0.29 \mu \mathrm{m}$ diameters, and in the JEOL 2010FX, using an aperture which selects a region of $\sim 0.18 \mu \mathrm{m}$ diameter. The TEM foil thicknesses were determined using a contamination-spot-separation method ${ }^{22}$ in the JEOL 2010FX. X-ray diffraction (XRD) was conducted with an $18 \mathrm{~kW}$ Rigaku 300 rotating anode source with a $\mathrm{Cu}$ target and a $\mathrm{MgO}(100)$ monochromator. $\theta-2 \theta$ scans were carried out first to locate the peaks associated with potential new phases, and x-ray rocking curves (XRCs) were subsequently performed for the purpose of maximizing the diffraction intensity and determining the peak positions accurately. The PL experiments were performed on samples cooled to $7.5 \mathrm{~K}$ in a helium gas flow and photoexcited with both the $632 \mathrm{~nm}$ line of a $\mathrm{He}-\mathrm{Ne}$ laser and the $325 \mathrm{~nm}$ line of a $\mathrm{He}-\mathrm{Cd}$ laser.

\section{RESULTS AND DISCUSSION}

\section{A. Structural evolution}

Figure 1(a) shows the $\theta-2 \theta$ x-ray scans of un-implanted, as-implanted, and implanted-plus-annealed samples. In the as-implanted and implanted-plus-annealed samples, peaks at $2 \theta \approx 86^{\circ}$ are apparent. These peaks may correspond to $\mathrm{ZB}$ GaN $\{004\}$, which has a $2 \theta$ value of $86.7^{\circ} .{ }^{23,24}$ Preliminary information about these layers may be estimated via the full width at half maxima (FWHM) of the peaks. Assuming that the sample contains a random distribution of strain-free crystalline regions of dimension smaller than $\sim 100 \mathrm{~nm}$, the crystal dimension is given by the Scherrer equation as $L$ $=[0.89 \lambda / B(2 \theta) \cos \theta]$, where $\theta$ is the Bragg angle, $B(2 \theta)$ is the FWHM in $2 \theta$, and $\lambda$ is the x-ray wavelength. ${ }^{25}$ Thus, the dimensions of the new phases are estimated as approximately $3 \mathrm{~nm}$ for both the as-implanted and implanted-plusannealed samples. We note that the Scherrer equation provides an estimate of the crystallite sizes; additional information about the shapes and dimensions of these new crystalline phases has been determined using TEM, as described below.

To determine more precise values of the $d$-spacings of these new crystalline phases, we performed XRCs. Figure 1(b) shows (004) GaAs XRCs for the as-implanted and implanted-plus-annealed samples. The presence of (004) GaN peaks in (004) GaAs XRCs suggests a preferential alignment of the (004) planes of the GaN-rich phases with
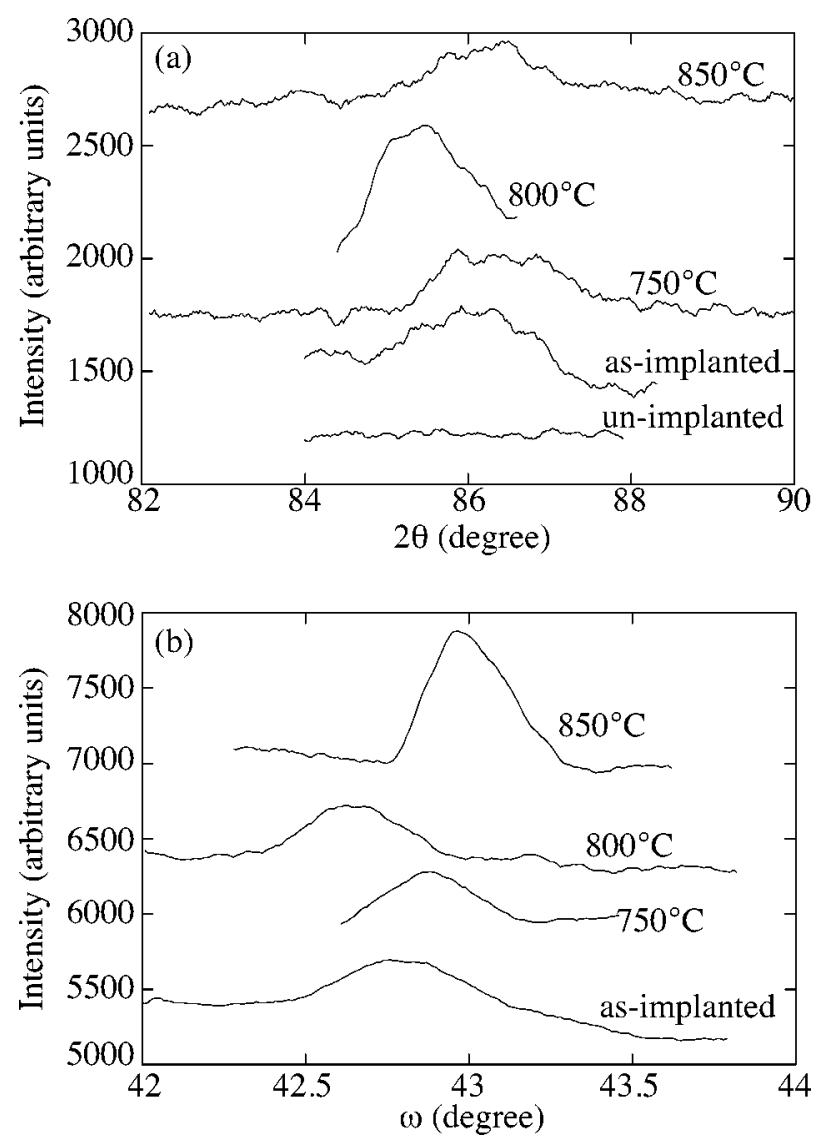

FIG. 1. (a) $\theta-2 \theta$ x-ray scans and (b) XRCs of the un-implanted, asimplanted, and implanted-plus-annealed samples. 
respect to GaAs. From these XRCs, we determined interplanar spacings of $1.136 \pm 0.001,1.135 \pm 0.001,1.140 \pm 0.001$, and $1.133 \pm 0.001 \AA$ for as-implanted, 750,800 , and $850{ }^{\circ} \mathrm{C}$ annealed samples, respectively, within $1.3 \%$ of the $\{004\}$ interplanar spacing of ZB GaN, $1.125 \AA^{23,24}$

To further examine the structure of the nanometer-sized phases, we employed diffraction contrast TEM. Figure 2 shows dark-field diffraction contrast TEM images of (a) asimplanted and (c) 750 , (e) 800 , and (g) $850^{\circ} \mathrm{C}$ annealed samples. These dark-field images were obtained using the GaAs 004 beam and when possible, a portion of the diffraction ring closest to the GaAs 004 spot. The corresponding SAD patterns, shown in Figs. 2(b), 2(d), 2(f), and 2(h), respectively, were collected in the JEOL 4000EX, using an aperture which selects an area with a diameter of $\sim 0.29 \mu \mathrm{m}$, and thus includes all three layers. Most of the TEM images show evidence of three regions: (1) a surface layer, (2) $\sim 150$-nm-thick middle layer, and (3) a near-substrate layer.

For the as-implanted sample shown in Fig. 2(a), the middle layer appears opaque, suggesting that it is essentially amorphous. This is also indicated by the SAD pattern shown in Fig. 2(b), which contains both diffraction spots associated with $\mathrm{ZB} \mathrm{GaAs}$, and a diffuse ring presumably due to the apparently amorphous middle layer. ${ }^{26,27}$ However, our highresolution images, which are presented below, suggest the presence of small $(<2.5 \mathrm{~nm})$ crystallites in the middle layers of the as-implanted sample.

For the annealed samples shown in Figs. 2(c), 2(e), and $2(\mathrm{~g})$, the middle layers contain a high density of $2-10 \mathrm{~nm}$ sized circular bright features, suggesting the formation of nanometer-sized crystallites. The corresponding SAD patterns in Figs. 2(d), 2(f), and 2(h) contain the ZB GaAs spots and the diffuse ring, plus additional spotty rings due to crystalline regions with various orientations. Using the GaAs diffraction spots as an internal standard, we determined the interplanar spacings corresponding to those rings. For example, for the three rings shown in each diffraction pattern of the annealed samples, the corresponding interplanar spacings are $2.61 \pm 0.01,1.60 \pm 0.01$, and $1.37 \pm 0.01 \AA$, respectively. They are similar to $\{111\},\{220\}$, and $\{113\}$ interplanar $d$ spacings of pure $\mathrm{ZB} \mathrm{GaN}$, which are $2.60,1.59$, and 1.36 $\AA$, respectively. ${ }^{23,24}$

Close examination of the diffraction patterns in Fig. 2 reveals that the GaN-related diffraction rings appear spotty, with orientation-dependent intensity variations. For the $\{111\}$ and $\{220\}$ rings of the annealed samples, segments of the rings have higher intensity, suggesting the presence of preferred crystallographic orientations. In Figs. 2(d), 2(f), and $2(\mathrm{~h})$, the segments of the $\{111\}$ and $\{220\}$ diffraction rings marked with arrows suggest significant $\{111\}_{\text {crystallite }} / /\{111\}_{\mathrm{GaAs}}$ and $\{220\}_{\text {crystallite }} / /\{220\}_{\mathrm{GaAs}}$ preferential alignments between the GaAs substrate and $\mathrm{GaN}$ crystallites. We also collected SAD patterns from individual layers and combinations of two layers, using SAD apertures which select areas with 0.18 and $0.14 \mu \mathrm{m}$ diameters. The additional SAD patterns suggest that the $\{111\}$ texture is strongest in the vicinity of the interfaces between the middle layer and the surface and near-substrate layers. Furthermore, the SAD patterns show evidence for weak
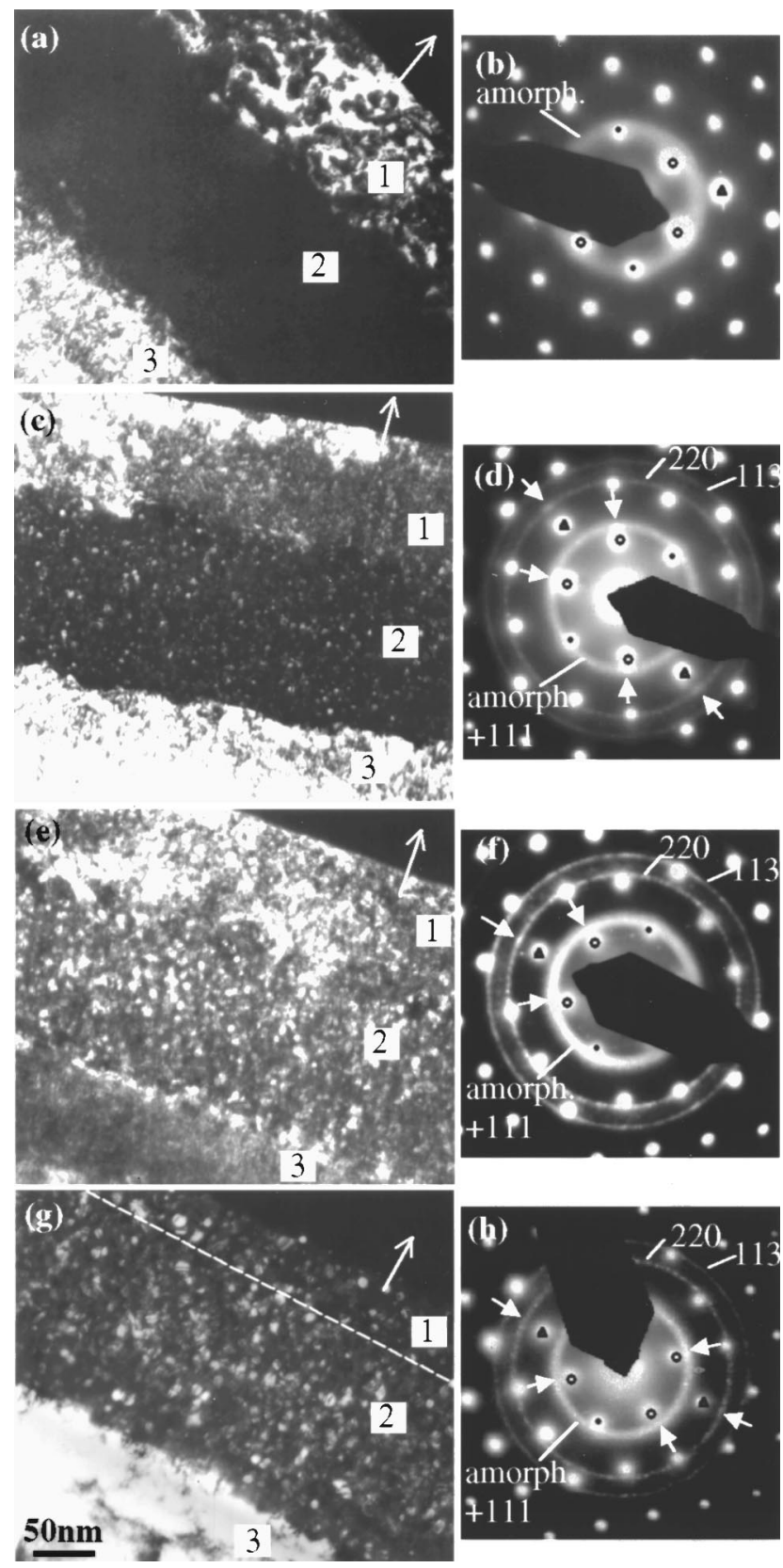

FIG. 2. Dark-field diffraction contrast TEM images of (a) as-implanted sample and samples annealed at (c) 750, (e) 800, and (g) $850{ }^{\circ} \mathrm{C}$, obtained using the GaAs $\{004\}$ beam, and when possible, a portion of the crystallite $\{113\}$ diffraction ring closest to the GaAs $\{004\}$ spot. The direction of the arrow in each figure is $\langle 004\rangle$. (b), (d), (f), and (h) are the SAD patterns corresponding to $(\mathrm{a}),(\mathrm{c}),(\mathrm{e})$, and $(\mathrm{g})$, respectively. The major diffraction spots from the crystalline GaAs $\{111\},\{220\}$, and $\{002\}$, are indicated by circles, triangles, and black dots, respectively. In (g), approximately two thirds of the surface layer has cleaved off. The remaining part of the surface layer has a microstructure similar to that of the middle layer. The dashed line indicates the approximate position of the interface between the remaining surface layer and the middle layer. The regions labeled 1, 2, and 3 are described in the text.

$\{004\}_{\text {crystallite }} / /\{004\}_{\text {GaAs }}$ preferential alignment. The stronger $\{004\}_{\text {crystallite }} / /\{004\}_{\text {GaAs }}$ alignment suggested by the XRCs shown in Fig. 1(b) is likely due to the fact that the $\{004\}$ XRCs selectively probe those $\{004\}$ planes, which are parallel to the $\{004\}$ GaAs planes.

We further examined the nanometer-scale structure of the crystallites using HRTEM. In Fig. 3(a), the seemingly 

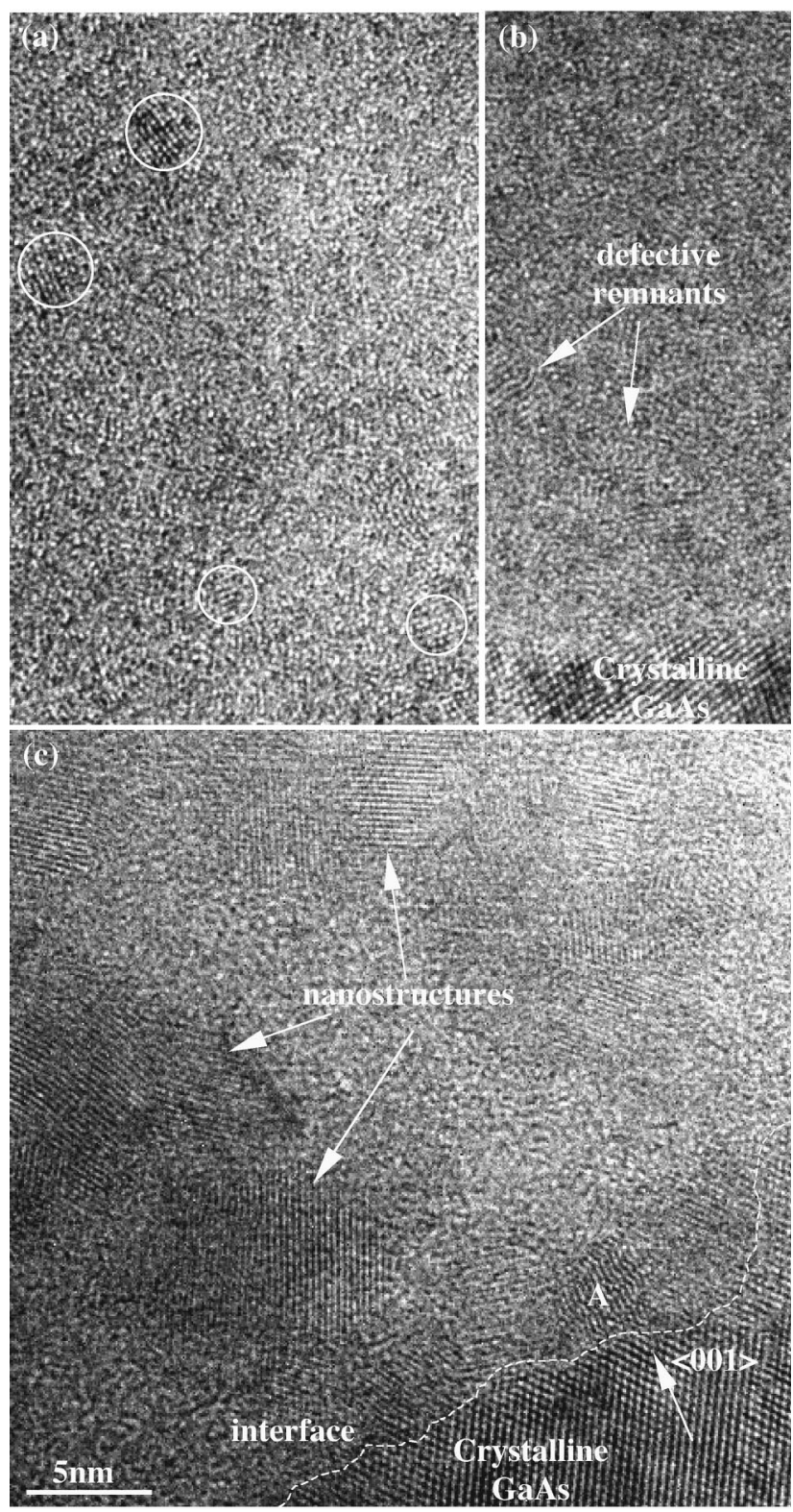

FIG. 3. HRTEM images in the N-implanted GaAs samples: (a) the middle layer of the as-implanted sample, with small crystallites circled, (b) interface between the middle and near-substrate layers, with defective GaAs remnants indicated by arrows, and (c) HRTEM image in the $800{ }^{\circ} \mathrm{C}$ annealed sample, near the interface between the middle and near-substrate layers, with crystallites indicated by arrows. In all cases, the zone axis is $\langle 110\rangle$.

amorphous matrix of the as-implanted samples contains essentially defect-free crystallites with diameters $<2.5 \mathrm{~nm}$. As suggested by the XRD data, these crystallites are likely GaNrich phases formed during the elevated temperature implantation process. In the implanted-plus-annealed samples, similar crystallites, with significantly larger sizes, typically 2-10 $\mathrm{nm}$, were observed, an example of which is shown in Fig. 3(c), for the $800^{\circ} \mathrm{C}$ annealed sample.

In both cases, the presence of GaN-rich phases is further supported by the average lattice fringe spacings in the crystallites, $2.64 \pm 0.01 \AA$, within $3.5 \%$ of the $\{111\}$ interplanar spacing of ZB GaN, $2.61 \AA$. The apparently larger than bulk lattice spacings may be due to a slight tensile distortion of the planes in the crystallite, or due to the incorporation of
$4 \pm 1 \%$ As into GaN. The presence of GaN-rich crystallites in an amorphous matrix indicates an easier nucleation of $\mathrm{ZB}$ $\mathrm{GaN}$ than $\mathrm{ZB}$ GaAs. We are currently investigating the origin of the stronger driving force for the formation of $\mathrm{ZB} \mathrm{GaN}$ versus $\mathrm{ZB}$ GaAs.

In the as-implanted sample, defective crystallites with diameters of $\sim 5 \mathrm{~nm}$ were occasionally observed near the interface between the middle and near-substrate layers. Examples of defective crystallites are shown in Fig. 3(b). Interestingly, the average lattice fringe spacings of the defective crystallites are $3.25 \pm 0.05 \AA$, within $0.5 \%$ of the $\{111\}$ interplanar spacing of ZB GaAs, $3.26 \AA$. These defective crystallites are likely remnants of the parent crystalline GaAs, similar to earlier studies of ion-irradiated GaAs. ${ }^{28}$

In the implanted-plus-annealed samples, defective crystallites occasionally appear near the interface between the middle and near-substrate layers. An example defective crystallite is shown as "A" in Fig. 3(c). Interestingly, the average lattice fringe spacings of the defective crystallites are 2.9 $\pm 0.1 \AA$, suggesting the presence of a GaAs-GaN alloy, presumably nucleated at "remnant" GaAs crystallites, such as those shown in Fig. 3(b). Furthermore, the close proximity of this defective crystallite to the near-substrate layer is likely due to the solid phase epitaxial (SPE) growth of crystalline GaAs from the near-substrate layer toward the defective crystallite. The SPE growth rate $v$ of GaAs amorphized by As ion implantation is

$$
v=2.5 \times 10^{7} \exp \left(-\frac{1.6 \mathrm{eV}}{k_{B} T}\right)(\AA / \mathrm{s}),
$$

where $k_{B}$ is the Boltzmann constant and $T$ is the absolute temperature. ${ }^{29}$ For our sample annealed at $800{ }^{\circ} \mathrm{C}$ for $30 \mathrm{~s}$, we estimate $\sim 2.3 \mathrm{~nm}$ SPE regrowth of GaAs, which is consistent with the distance between the defective crystallite and the interface between the middle and near-substrate layers, as shown in Fig. 3(b).

It is interesting to note that the GaN-rich crystallites have apparently chosen to nucleate in the ZB form in a seemingly amorphous matrix, even though the $\mathrm{WZ}$ form of $\mathrm{GaN}$ is most stable thermodynamically. ${ }^{30}$ The preference for nucleation of $\mathrm{ZB} \mathrm{GaN} \mathrm{might} \mathrm{be} \mathrm{related} \mathrm{to} \mathrm{the} \mathrm{strain} \mathrm{present}$ in the crystallites. For example, a computational study predicted that the $\mathrm{ZB}$ form of $\mathrm{GaN}$ is most stable near a strained GaN/GaAs interface. ${ }^{31}$ Another possibility is that the small size of the GaN nanocrystallites may affect the relative stability of the ZB vs the $\mathrm{WZ}$ form. ${ }^{32}$ In the CdS system, it has been shown that as the size of nanoparticles decreases, the equilibrium crystal structure transforms from $\mathrm{WZ}$ to $\mathrm{ZB}$. This was explained by a minimization of surface free energy per unit volume, via the adoption of the ZB crystal structure, which has a higher density of low energy $\{111\}$ planes on the surface, in comparison with the WZ structure. Finally, the apparently amorphous matrix may possess some short-tomedium range order, which influences the structure of the crystallites. In particular, it is possible that the majority of the $\mathrm{Ga}$ and As atoms retain their ZB tetrahedral coordination, and some of them may act as the crystal nucleation site. Indeed, evidence of such short-range order has been found in GaAs amorphized by $\mathrm{Ga}$ and As co-implantation. ${ }^{33}$ We are 
currently endeavoring to use fluctuation microscopy ${ }^{34}$ to obtain further information regarding the structure of the crystallites and any medium-range order which may be present in the apparently amorphous matrix.

\section{B. Mechanisms of crystallite coarsening}

In order to gain insight into the mechanisms of crystallite coarsening, we investigated the evolution of the crystallite sizes with annealing temperature. For this purpose, we quantified distributions of crystallite sizes, using an analysis of several high-resolution TEM images. The crystallite size distribution analysis procedure is described in the Appendix of this article. We also quantified the crystallite volume fractions $V_{f}$ using these distributions in conjunction with TEM foil thickness measurements.

Figure 4(a) shows the distributions of crystallite sizes, determined from several HRTEM images, and fit with a Gaussian. With increasing annealing temperature, the average crystallite sizes become larger, while the volume fraction of crystallites is consistently approximately $2 \%$. The apparently mass-conservative crystallite coarsening with increasing temperature suggests that the crystallization and precipitation processes are essentially complete. Instead, Ostwald ripening is likely the dominant process governing the coarsening. ${ }^{35,36}$ This is further confirmed by the selfsimilarity of the normalized size distributions shown in Fig. 4(b), where the frequency $f$ is the number of crystallites with diameters within the specified range, divided by the total number of crystallites; $d$ is the crystallite diameter; and $d_{m}$ is the mean crystallite diameter.

Ostwald ripening involves atomic diffusion between precipitates, such that larger precipitates grow at the expense of smaller ones. ${ }^{35}$ To determine the activation energy for this process, we assume that the crystallites have a spherical shape, and apply the modified Lifshitz-Slyozov-Wagner (MLSW) theory. ${ }^{37,38}$ In the MLSW theory, the growth kinetics of spherical precipitates during particle coarsening are described as

$$
r_{m}^{3}-r_{0}^{3}=K \frac{D C_{\alpha}(\infty) t}{T}
$$

where, $r_{0}$ and $r_{m}$ are the average precipitate radii before and after the coarsening process, $K$ is a constant, $D$ is the solute diffusion coefficient in the matrix, $C_{\alpha}(\infty)$ is the equilibrium solute concentration in the matrix, $t$ is time, and $T$ is the absolute temperature. In our study, the annealing time is fixed. Therefore, in our case, assuming that the product $D C_{\alpha}(\infty)$ is proportional to $\exp \left(-E_{a} / k_{B} T\right)$ :

$$
T\left(d_{m}^{3}-d_{0}^{3}\right) \propto e^{-\left(E_{a} / k_{B} T\right)},
$$

where $d_{0}$ is the average crystallite diameter in the asimplanted sample, $E_{a}$ is the activation energy of the coarsening process, and $k_{B}$ is the Boltzmann constant. ${ }^{39}$ In Fig. $5(\mathrm{a})$, we plot $T\left(d_{m}^{3}-d_{0}^{3}\right)$ versus the reciprocal annealing temperature $1 / T$. The $d_{0}$ value was determined from the distribution of crystallite sizes obtained from HRTEM images, shown in Fig. 5(b), while the $d_{m}$ values were obtained from the crystallite size distributions in Fig. 4. A linear leastsquare fitting of the data in Fig. 5(a) leads to an activation
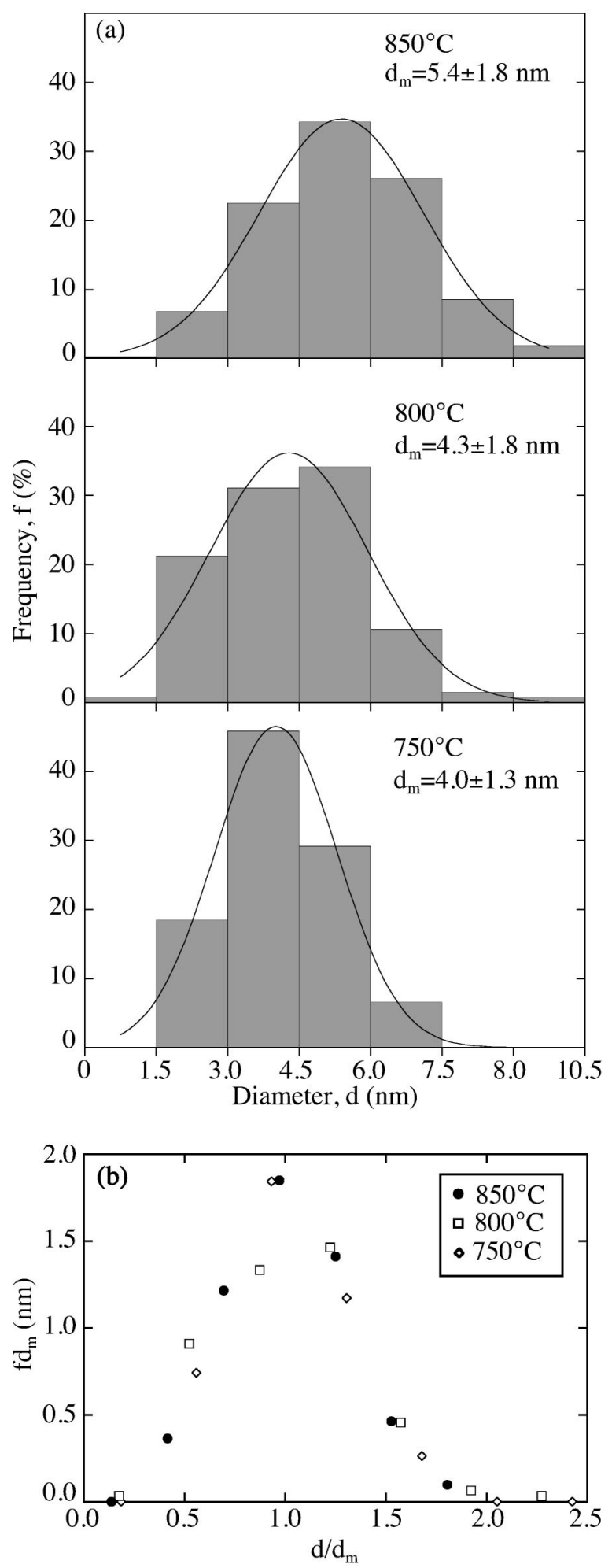

FIG. 4. (a) Size distribution and (b) normalized size distribution of the crystallites in implanted-plus-annealed samples, determined from an analysis of HRTEM images.

energy of $1.0 \pm 0.3 \mathrm{eV}$ for this diffusion-controlled Ostwald ripening process. In an earlier study, an activation energy of $3.6 \mathrm{eV}$ was reported for nitrogen diffusion in a crystalline GaAs matrix, which was apparently governed by As interstitials. ${ }^{40}$ It is possible that the increased defect density of our apparently amorphous GaAs matrix in comparison with its crystalline counterpart, might lead to a significantly 

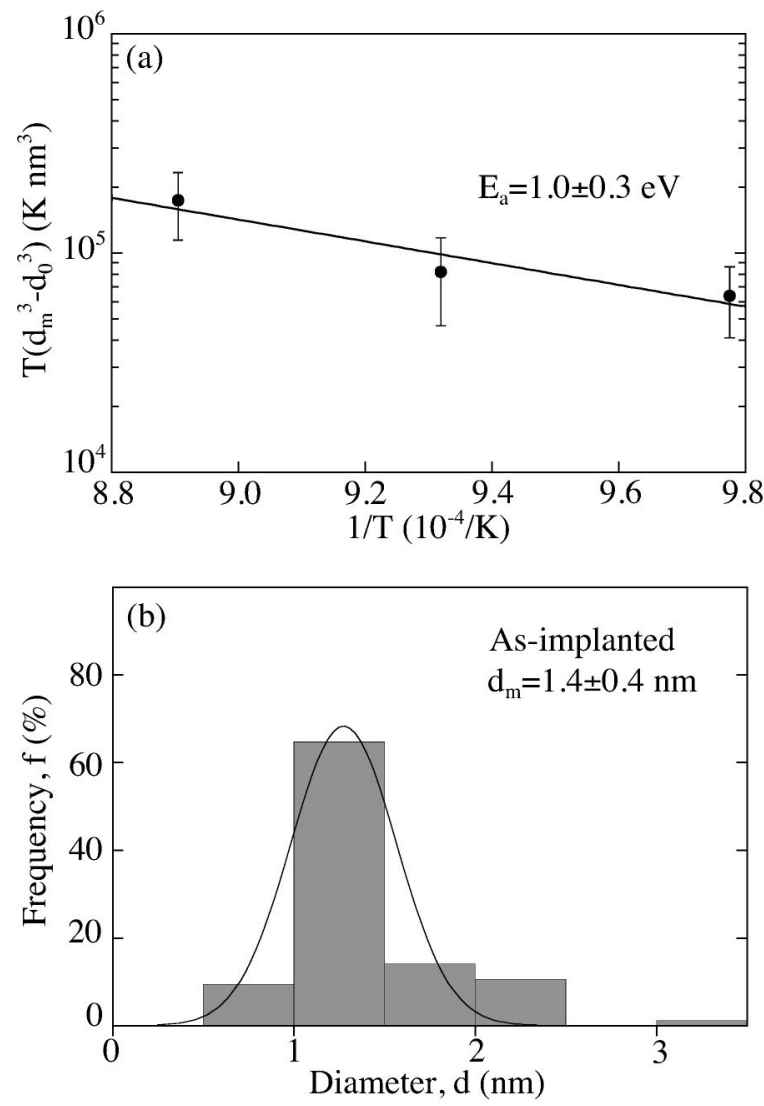

FIG. 5. (a) A plot of the logarithm of $T\left(d_{m}^{3}-d_{0}^{3}\right)$ vs the reciprocal annealing temperature $1 / T$. The $d_{m}$ and $d_{0}$ values were determined from highresolution images, and the line represents a linear least-square fit to the data. (b) Crystallite size distribution of the as-implanted sample determined from an analysis of HRTEM images.

lower activation energy for diffusion of nitrogen in GaAs. Interestingly, the activation energy for As precipitate growth in low-temperature-grown GaAs was reported as $1.4 \pm 0.3$ $\mathrm{eV}$, and attributed to enhanced diffusion of As due to the supersaturation of $\mathrm{Ga}$ vacancies in low-temperature-grown GaAs. ${ }^{39}$ A comparison of these activation energies suggests that our crystallite coarsening process may be governed by $\mathrm{N}$ or As diffusion via gallium vacancies.

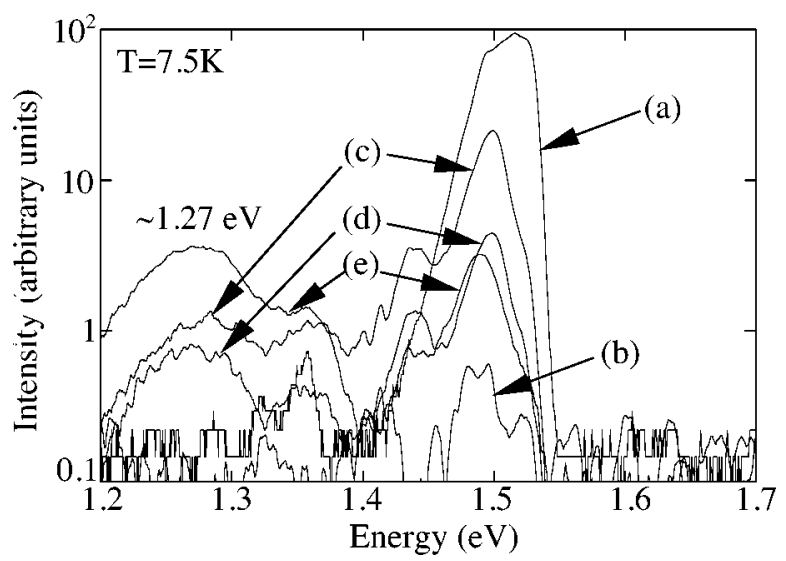

FIG. 6. Photoluminescence spectroscopy of the (a) un-implanted, (b) asimplanted, (c) $750{ }^{\circ} \mathrm{C}$ annealed, (d) $800{ }^{\circ} \mathrm{C}$ annealed, and (e) $850{ }^{\circ} \mathrm{C}$ annealed samples.

\section{Optical properties}

We examined both the short- and long-wavelength optical properties of the samples. PL spectral features were not observed in the short-wavelength range. Instead, we determined that the feature apparently observed at $3.3 \mathrm{eV}$ in Ref. 17 was likely an artifact arising from the Lorentzian tail of the $325 \mathrm{~nm}$ line of the $\mathrm{He}-\mathrm{Cd}$ laser, cutoff to short wavelengths by an optical filter. Figure 6 shows the longwavelength PL spectra of (a) un-implanted, (b) as-implanted, and (c)-(e) implanted-plus-annealed samples. Most of these PL spectra are dominated by a strong emission near $1.5 \mathrm{eV}$, due to the fundamental band gap transition of GaAs. In the spectrum of Fig. 6(b), the $1.5 \mathrm{eV}$ emission is significantly quenched, presumably due to the amorphization of the GaAs during the ion implantation process. In the annealed samples, three additional emissions are apparent. The emissions near $1.44 \mathrm{eV}$ are likely related to $\mathrm{Ga}_{\mathrm{As}}$, similar to an earlier report of GaAs annealed in a Ga-rich atmosphere. ${ }^{41}$ The Ga-rich atmosphere is likely to lead to a GaAs surface decomposition similar to the As-poor atmosphere present during our uncapped RTA process. The emissions near $1.35 \mathrm{eV}$ are likely due to a $\mathrm{V}_{\mathrm{As}}-\mathrm{C}$ complex, similar to an earlier report of epitaxially grown GaAs. ${ }^{42}$ The emissions near $1.27 \mathrm{eV}$ are similar to those observed in earlier ion-beam-synthesis studies of GaAs:N. In those cases, the emissions were attributed to the fundamental band gap transitions of dilute GaNAs alloys. ${ }^{12,13,16}$ However, the presence of the As-rich GaAsN alloys was not confirmed by an analysis of the nanometerscale structure.

Our experiments suggest instead that this emission is related to the formation of GaN-rich crystallites. Assuming that the $1.27 \mathrm{eV}$ emission corresponds to a fundamental band gap transition, the band gap energy of these GaN-rich nanostructures is significantly smaller than that of bulk ZB GaN $(3.3 \mathrm{eV}) .{ }^{43}$ An earlier report predicted strain-induced lowering of the $\mathrm{GaN}$ band gap from $3.5 \mathrm{eV}$ to $\sim 0.5$ to $1.7 \mathrm{eV}$ for a fully coherently strained $\mathrm{GaN}$ cluster in a GaAs matrix. ${ }^{8} \mathrm{In}$ order to lower the GaN bandgap to $\sim 1.27 \mathrm{eV}$, a residual strain of $>8 \%$ would be needed. Our XRD and TEM data suggest that the crystallite lattice parameters are within $1.4 \%$ and $1.6 \%$ of those of pure $\mathrm{ZB} \mathrm{GaN}$. Thus, strain is not likely to be the mechanism of band gap lowering. On the other hand, the incorporation of $\sim 4$ at. \% As into GaN is predicted to lower the band gap to $\sim 1.27 \mathrm{eV}^{2}$. Using a linear interpolation of GaAs and GaN lattice parameters, this corresponds to an alloy, $\mathrm{GaAs}_{0.08} \mathrm{~N}_{0.92}$, with a lattice parameter of $4.59 \AA$, within $2.0 \%$ of our electron diffraction results and $1.3 \%$ of our x-ray diffraction results. Therefore, the incorporation of As into GaN is a likely explanation for the apparent band gap lowering. Furthermore, the slight blueshift of the observed emission with decreasing average crystallite size suggests the possibility of quantum confinement in the GaAsN nanostructures. Further work is in progress to examine this effect.

\section{CONCLUSIONS}

In summary, we have synthesized GaAsN nanostructures by nitrogen ion implantation into epitaxial GaAs films and subsequent rapid thermal annealing. These nanometer-sized 
crystallites possess symmetry and lattice parameters similar to that of $\mathrm{ZB} \mathrm{GaN}$. The average crystallite size increases with annealing temperature, while the size distributions are self-similar and the volume fraction remains constant. Thus, the crystallite coarsening process is considered to be governed by Ostwald ripening, with an activation energy of 1.0 $\mathrm{eV}$. A comparison of this activation energy with earlier studies suggests that our coarsening process may be governed by $\mathrm{N}$ or As diffusion via gallium vacancies. Photoluminescence spectroscopy shows a significant emission at $\sim 1.27 \mathrm{eV}$ for the implanted-plus-annealed samples, apparently related to the GaAsN nanostructures. The lowering of the fundamental band gap of these crystallites is likely due to the incorporation of $\mathrm{As}$ in $\mathrm{GaN}$.

\section{ACKNOWLEDGMENTS}

This work was supported in part by the U-M Office of the Vice President for Research Initiative in Materials Research Grant; the DoD Multidisciplinary University Research Initiative (MURI), administered by the Air Force Office of Scientific Research under Grant No. F49620-00-10328); the Department of Energy, through the National Renewable Energy Laboratory Photovoltaics Beyond the Horizon Program under Contract No. ACQ-1-30619-14, and the TRW Foundation. S. J. C. was supported in part by the NSFREU Program through Grant No. 9773707, and by scholarships from the Marian Sarah Parker Scholars and the General Electric Faculty for the Future Programs. We thank P. Smereka and J. Kieffer for useful discussions, L. R. Berry for assistance with PROFILE code simulations, and L. Murfey and A. Bhardwaj for assistance with TEM sample preparation. The authors also acknowledge the assistance of the staff of the Michigan Ion Beam and the Electron Microbeam Analysis Laboratories (EMAL) at the University of Michigan. The JEOL 2010FX analytical electron microscope at EMAL is supported by NSF Grant No. 9871177.

\section{APPENDIX}

We quantified distributions of crystallite sizes using HRTEM images as follows. First, for each HRTEM image, we performed fast Fourier transforms (FFTs), which resulted in spotty $\{111\}$ reflections associated with GaN-rich crystallites. For those images showing part of the GaAs nearsubstrate region, reflections of GaAs were also apparent. Next, we selected conjugate pairs of $\{111\} \mathrm{GaN}$ reflection spots in the FFT to produce Fourier-filtered images (inverse FFTs) of individual crystallites. For each filtered image, pixels within the top $25 \pm 5 \%$ of the total grayscale range were considered to make up the crystallites. Assuming spherical crystallites, we then calculated the crystallite sizes (diameters) and quantified the size distributions for samples annealed at different temperatures.

\footnotetext{
${ }^{1}$ I. A. Buyanova, W. M. Chen, and B. Monemar, MRS Internet J. Nitride Semicond. Res. 6, 1 (2001).

${ }^{2}$ L. Bellaiche, S.-H. Wei, and A. Zunger, Phys. Rev. B 54, 17568 (1996).

${ }^{3}$ W. G. Bi and C. W. Tu, Appl. Phys. Lett. 70, 1608 (1997).

${ }^{4}$ Y. Zhao, F. Deng, S. S. Lau, and C. W. Tu, J. Vac. Sci. Technol. B 16, 1297 (1998).
}

${ }^{5}$ K. Uesugi, N. Morooka, and I. Suemune, Appl. Phys. Lett. 74, 1254 (1999).

${ }^{6}$ J. Salzman and H. Temkin, Mater. Sci. Eng., B 50, 148 (1997).

${ }^{7}$ R. S. Goldman, R. M. Feenstra, B. G. Briner, M. L. O'Steen, and R. J. Hauenstein, Appl. Phys. Lett. 69, 3698 (1996).

${ }^{8}$ R. S. Goldman, R. M. Feenstra, B. G. Briner, M. L. O'Steen, and R. J. Hauenstein, J. Electron. Mater. 26, 1997 (1997).

${ }^{9}$ H. P. Xin, K. L. Kavanagh, Z. Q. Zhu, and C. W. Tu, Appl. Phys. Lett. 74, 2337 (1999).

${ }^{10}$ T. S. Cheng, C. T. Foxon, L. C. Jenkins, S. E. Hooper, J. W. Orton, S. V. Novikov, T. B. Popova, and V. V. Tretyakov, J. Cryst. Growth 158, 399 (1996).

${ }^{11}$ G. Mendoza-Diaz, K. S. Stevens, A. F. Schwartzman, and R. Beresford, J. Cryst. Growth 178, 45 (1997).

${ }^{12}$ W. Shan, K. M. Yu, W. Walukiewicz, J. W. Ager III, E. E. Haller, and M. C. Ridgway, Appl. Phys. Lett. 75, 1410 (1999).

${ }^{13}$ K. M. Yu, W. Walukiewicz, W. Shan, J. Wu, J. W. Beeman, J. W. Ager III, and E. E. Haller, Appl. Phys. Lett. 77, 3607 (2000).

${ }^{14}$ J. Jasinski, K. M. Yu, W. Walukiewicz, J. Washburn, and Z. LilientalWeber, Appl. Phys. Lett. 79, 931 (2001).

${ }^{15}$ K. M. Yu, W. Walukiewicz, J. Wu, J. W. Beeman, J. W. Ager III, E. E. Haller, W. Shan, H. P. Xin, C. W. Tu, and M. C. Ridgway, J. Appl. Phys. 90, 2227 (2001).

${ }^{16}$ T. Shima, S. Kimura, T. Iida, A. Obara, Y. Makita, K. Kudo, and K. Tanaka, Nucl. Instrum. Methods Phys. Res. B 118, 743 (1996).

${ }^{17}$ K. Kuriyama, T. Tsunoda, N. Hayashi, and Y. Takahashi, Nucl. Instrum. Methods Phys. Res. B 148, 432 (1999).

${ }^{18}$ X. W. Lin, M. Behar, R. Maltez, W. Swider, Z. Liliental-Weber, and J. Washburn, Appl. Phys. Lett. 67, 2699 (1995).

${ }^{19}$ Implantation Science Corp., Wakefield, MA 01880.

${ }^{20} \mathrm{~J}$. Ziegler, IBM Corp.

${ }^{21}$ G. Amsel, J. P. Nadai, E. d'Artemare, D. David, E. Girard, and J. Moulin, Nucl. Instrum. Methods 92, 481 (1971).

${ }^{22}$ D. B. Williams, Practical Analytical Electron Microscopy in Materials Science (Philips Electronic Instruments, Mahwah, 1984).

${ }^{23}$ H. Okumura, S. Misawa, and S. Yoshida, Appl. Phys. Lett. 59, 1058 (1991).

${ }^{24}$ S. Strite, J. Ruan, Z. Li, A. Salvador, H. Chen, D. J. Smith, W. J. Choyke, and H. Morkoç, J. Vac. Sci. Technol. B 9, 1924 (1991).

${ }^{25}$ B. E. Warren, X-ray Diffraction (Dover, New York, 1990).

${ }^{26}$ X. Yang, R. Wang, H. Yan, and Z. Zhang, Mater. Sci. Eng., B 49, 5 (1997).

${ }^{27}$ I. Jenčič, M. W. Bench, I. M. Robertson, and M. A. Kirk, J. Appl. Phys. 69, 1287 (1991).

${ }^{28}$ B. A. Turkot, D. V. Forbes, I. M. Robertson, J. J. Coleman, L. E. Rehn, M. A. Kirk, and P. M. Baldo, J. Appl. Phys. 78, 97 (1995).

${ }^{29}$ C. Licoppe, Y. Nissim, C. Meriadec, and P. Hénoc, Appl. Phys. Lett. 50, 1648 (1987).

${ }^{30}$ M. Leszczynski, in Properties, Processing and Applications of Gallium Nitride and Related Semiconductors, edited by J. H. Edgar, S. T. Strite, I. Akasaki, H. Amano, and C. Wetzel (INSPEC, London, UK, 1999), p. 3.

${ }^{31}$ T. Ito, K. Shiraishi, and A. Taguchi, J. Cryst. Growth 227-228, 366 (2001).

${ }^{32}$ R. Banerjee, R. Jayakrishnan, and P. Ayyub, J. Phys.: Condens. Matter 12, 10647 (2000).

${ }^{33}$ M. C. Ridgway, C. J. Glover, G. Foran, and K. M. Yu, J. Appl. Phys. 83, 4610 (1998).

${ }^{34}$ M. M. J. Treacy and J. M. Gibson, Acta Crystallogr., Sect. A: Found. Crystallogr. 52, 212 (1996).

${ }^{35}$ P. W. Voorhees, J. Stat. Phys. 38, 231 (1985)

${ }^{36}$ Y. Masuda and R. Watanabe, Sintering Processes, Materials Science Research (Plenum, New York, 1979), Vol. 13.

${ }^{37}$ J. M. Martin and R. D. Doherty, Stability of Microstructure in Metallic Systems (Cambridge University Press, London, 1976).

${ }^{38}$ A. J. Ardell, Acta Metall. 20, 61 (1972).

${ }^{39}$ Z. Liliental-Weber, X. W. Lin, J. Washburn, and W. Schaff, Appl. Phys. Lett. 66, 2086 (1995).

${ }^{40}$ G. Bösker, N. A. Stolwijk, J. V. Thordson, U. Södervall, and T. G. Andersson, Phys. Rev. Lett. 81, 3443 (1998).

${ }^{41}$ N. H. Ky and F. K. Reinhart, J. Appl. Phys. 83, 718 (1998).

${ }^{42}$ N. H. Ky, D. Martin, and F. K. Reinhart, Physica B 273, 729 (1999).

${ }^{43}$ M. Leroux and B. Gil, in Properties, Processing and Applications of Gallium Nitride and Related Semiconductors, edited by J. H. Edgar, S. T. Strite, I. Akasaki, H. Amano, and C. Wetzel (INSPEC, London, UK, 1999), p. 45. 